\title{
Testing Pre-Main Sequence Models with Young Binaries
}

\author{
Francesco Palla \\ Osservatorio Astrofisico di Arcetri, Largo E. Fermi 5, 50125 Firenze, \\ Italy
}

\begin{abstract}
I will discuss several tests to gauge the accuracy of pre-mainsequence (PMS) models. Methods to determine the mass of young stars are overviewed, with emphasis on the information provided by doublelined, spectroscopic binary systems. A comparison of the dynamically determined masses with those estimated using the PMS models of Palla \& Stahler (1999) is presented. Good agreement between empirical and theoretical masses is found. The analysis of the inferred ages from the isochrones shows a remarkable coevality within each binary system. A complete assessment of the accuracy of PMS tracks needs the identification of eclipsing systems of low-mass.
\end{abstract}

\section{Tests of PMS Evolutionary Tracks}

Two basic parameters used in observational checks of theoretical calculations of stellar evolution are stellar masses and ages. Direct determinations of PMS masses do exist now thanks to the discovery of a (still small) sample of spectroscopic eclipsing systems among these stars. Stellar ages can only be estimated indirectly through the use of evolutionary tracks and isochrones in the HR diagram. However, the actual pattern of stellar births within any given region offers an important clue for testing star formation theories.

PMS models are routinely produced by many groups, both in Europe and elsewhere. How are we to gauge the accuracy of PMS tracks? Several tests can be performed to this end, including the following ones:

- Position of the birthline: the observed upper envelope of the distribution of PMS in both $\mathrm{T}$ associations and groups containing Herbig Ae/Be stars does seem to match the predicted birthline (e.g. van den Ancker et al. 1998; Luhman 1999; Palla \& Stahler 2000a). Also, the predicted endpoint of the birthline at $\sim 8 \mathrm{M}_{\odot}$ appears supported by the observational data. Examples include the Trapezium cluster (Hillenbrand 1997), NGC 6611 (Hillenbrand et al. 1993), and a number of galactic and extragalactic OB associations (Massey et al. 1995; Massey \& Hunter 1998). These results give credit to the essential validity of protostar theory, leading to the birthline mass-radius relation.

- Pre-Main-Sequence masses and ages: the study of PMS binaries offers the best way to test the evolutionary tracks: the measurement of the dynamical mass of the components of any binary system yields in fact an absolute mass calibration of the tracks. Under the assumption of coeval formation, the derived 
ages of the components test relative age calibrations of the tracks. We will provide specific examples of the known systems and discuss the implications on current models.

- Lithium abundance: the observed surface abundances in low- and very-low mass stars provide a critical test for the physical processes (convection, opacity, rotation, mass loss) which ought to be included in the theoretical models in order to reproduce the observed level of depletion. Due to its small binding energy, ${ }^{7} \mathrm{Li}$ (the most abundant of the two stable isotopes of lithium) is easily destroyed in stellar interiors via the reaction ${ }^{7} \mathrm{Li}(\mathrm{p}, \alpha){ }^{4} \mathrm{He}$ at temperatures around $2.5 \times 10^{6} \mathrm{~K}$ (Bodenheimer 1965). According to standard models, depletion occurs only when the outer convection extends down to that temperature. Thus, it appears quite unavoidable that some ${ }^{7} \mathrm{Li}$ depletion due to nuclear burning takes place already during PMS contraction. Other processes, associated with the mixing due to transport of angular momentum through the star, are effective on much longer time scales and dominate the depletion history during the main sequence phase (e.g. Martín \& Claret 1996; Ventura et al. 1998). From the numerical point of view, the situation is still unsettled because of the strong sensitivity of the amount of lithium destruction on the temperature at the bottom of the convection zone, whose exact location is sensitive to opacity, convection and rotation (see Bildsten et al. 1997 for a different approach). On the positive side, there is the remarkable application of the theory of Li-burning to the search and discovery of brown dwarfs (Rebolo et al. '1996; Basri et al. 1996).

- Main-Sequence Binaries: these systems have provided the most accurate estimates of stellar masses and radii (e.g. Andersen 1991). For detached, doublelined, eclipsing systems, the radial velocity and light curves yield mass estimates of individual components, plus their luminosities and effective tempartures. Thus, their location in the HR diagram is a powerful test for the tracks. This is particularly important for low-mass systems $\left(\mathrm{M}_{*} \lesssim 0.6 \mathrm{M}_{\odot}\right.$, or $\left.\mathrm{T}_{\text {eff }} \lesssim 4000 \mathrm{~K}\right)$, for which the theoretical models are most uncertain due to the limited knowledge of low-temparture opacities, convection and model atmospheres. Unfortunately, selection effects render the discovery of eclipses in intrinsically faint and small stars rather difficult. The two known eclipsing binary systems are YY Gem $\left(M_{1,2}=0.62,0.57 \mathrm{M}_{\odot}\right)$ and $\mathrm{CM}$ Dra $\left(M_{1,2}=0.23,0.21 \mathrm{M}_{\odot}\right)$. Chabrier \& Baraffe (1995) have presented evolutionary models that reproduce the observed masses, radii and luminosities of both binary systems. A third system, GJ 2609 (a nearby M3.5 V quadruple system), has been recently discovered with individual masses of $\sim 0.4 \mathrm{M}_{\odot}$ (Delfosse et al. 1999). Unfortunately, no estimates of the stellar parameters are yet available.

- Pleiades Main-Sequence and Age: this cluster should provide the best test for the exact location of the ZAMS. However, the distance modulus (DM=5.32 mag or $116 \pm 3 \mathrm{pc}$; van Leeuwen \& Ruiz 1997) derived using the mean of the Hipparcos parallaxes is almost 0.3 mag smaller than that derived using the main-sequence fitting technique (Pinsonneault et al. 1998). This conflict has potential impact on a number of issues, the calibration in the extragalactic distance scale being the critical one. However, according to D'Antona \& Mazzitelli (1997), much of 
the discrepancy can be removed by adopting better effective temperature scales,

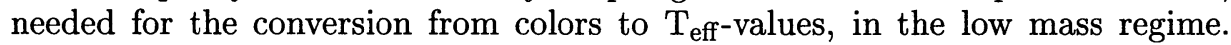

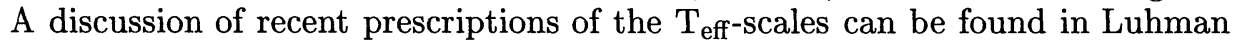
(1999; see also Baraffe in this volume).

\section{Estimating Stellar Masses: Single Stars}

Because of the central importance of obtaining reliable estimates of the mass of young stars, several methods have been employed.

- Surface gravities: One of the first methods utilizes the fundamental relation

$$
M_{*} / M_{\odot}=\frac{L_{*} g_{*} T_{e f f, \odot}^{4}}{L_{\odot} g_{\odot} T_{e f f, *}^{4}}
$$

where $M_{*}, L_{*}, g_{*}$ and $T_{\text {eff }}$ are the mass, luminosity, surface gravity and effective temperature of the program star. Spectrophotometric observations allowed McNamara (1976) to derive the masses of 36 stars of the Orion cluster. This technique is still used, particularly for high-mass stars. However, the precision of the method is rather poor.

- Infall velocities: Recently, Bonnell et al. (1998) have suggested an ingenious method that exploits the observed evidence of ongoing accretion in classical $\mathrm{T}$ Tauri stars. The infall speed of circumstellar matter traces directly the potential energy at the star surface. The assumption here is that the detected motion occurs along magnetic flux tubes that connect the star to the inner edge of the disk, $R_{i}$. The mass can then be evaluated from the relation

$$
M_{*}=\frac{V_{i}^{2} R_{*}}{2 G}\left(1-\frac{R_{*}}{R_{i}}\right)^{-1},
$$

where $V_{i}$ is the measured infall speed, $G$ the gravitational constant, and $R_{*}$ is the stellar radius. The latter can be calculated from the luminosity and effective temperature of the star. The ratio $R_{i} / R_{*}$ can be evaluated indirectly from the magnitude of the infrared excess (typically, $R_{i} / R_{*} \sim 4$ ). The method yields lower limits to the stellar mass because of projection effects and uncertainty in the assumption that the gas motion is indeed free-fall.

- Pulsational instabilities: Another potentially accurate technique is based on the pulsational properties of intermediate-mass stars. Stars with masses above $\sim 1 \mathrm{M}_{\odot}$ cross the instability strip in the HR diagram (Marconi \& Palla 1998). Several Herbig stars are now known to show periodic variability of the $\delta$ Scuti type, with periods less than $\sim 0 d_{3}$ and light amplitudes of $\sim 0.003-0.3 \mathrm{mag}$ (Marconi et al. 2000). Because of the sensitivity of pulsational instability models to the internal structure of the stars, the analysis of the observed characteristics can yield a strong constraint on their mass. This method will profit considerably from future space missions (such as MONS, COROT etc.) designed to study stellar oscillations in a large sample of stars, including young objects. This represents a powerful method for stars that are not part of the restricted group of spectroscopic binary systems. 
As an illustration, Figure 1 shows the location of HD 35929 in the HR diagram. The dotted box accounts for the uncertainty on the spectral type (A5 to F0) and distance ( $d=360$ to $430 \mathrm{pc}$; van den Ancker et al. 1998). The two circles indicate the best combination of the stellar parameters $\left(\mathrm{M}, \mathrm{L}, \mathrm{T}_{\text {eff }}\right)$ that yield a period equal to the observed one, $P=0.196 \pm 0.005 \mathrm{~d}$. The upper circle corresponds to a model of a $3.8 \mathrm{M}_{\odot}$ star with $\mathrm{L}=114 \mathrm{~L}_{\odot}$ and $\mathrm{T}_{\text {eff }}=$ $7100 \mathrm{~K}$ pulsating in the second overtone mode with $\mathrm{P}=0.198 \mathrm{~d}$. The lower circle represents the position of a $3.4 \mathrm{M}_{\odot}$ star with $\mathrm{L}=83 \mathrm{~L}_{\odot}$ and $\mathrm{T}_{\text {eff }}=7190 \mathrm{~K}$ pulsating in the first overtone mode with the same period. The two solutions indicate a mass of 3.4 or $3.8 \mathrm{M}_{\odot}$ : in both cases, HD 35929 can be considered a PMS pulsator, as expected.

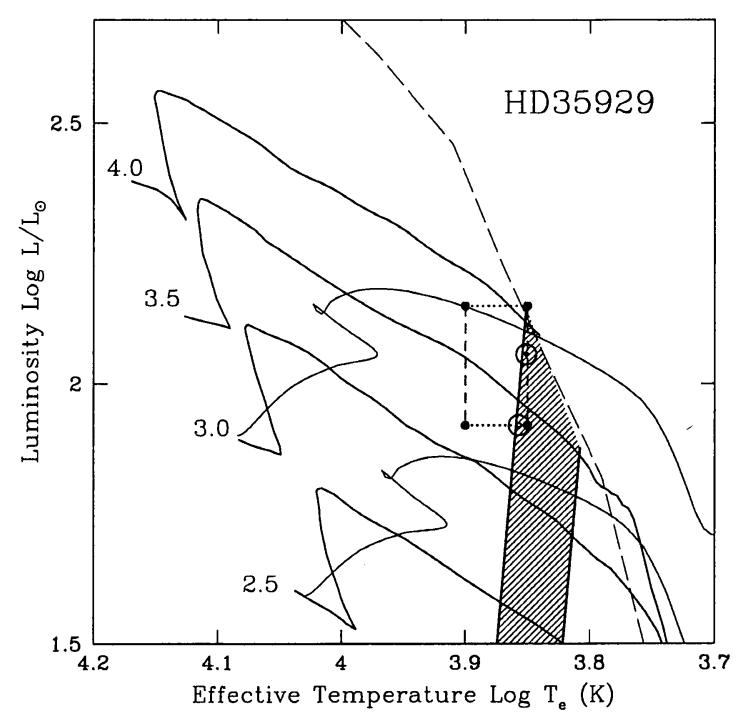

Figure 1. The position of HD 35929 in the HR diagram with the uncertainty on spectral type and distance indicated by the box. The instability strip is shown by the shaded region. The PMS and post-MS evolutionary tracks for 3.0 and $2.5 \mathrm{M}_{\odot}$ are shown as solid lines. (From Marconi et al. 2000)

\section{Estimating Stellar Masses: PMS Binary Stars}

The most reliable technique for determining stellar masses uses the observed rotational speeds. In this case, either spectroscopic binaries or circumstellar/circumbinary disks can be employed. Although the number of known PMS eclipsing binary systems (which yield individual masses) and double-lined spectroscopic systems (which give mass ratios) is still limited, it is possible to use the available information to gauge the accuracy of the tracks.

The initial results of the analysis of the available sample of PMS binaries are listed in Table 1 (from Palla \& Stahler 2000b). The observationally determined mass $\left(\mathrm{M}_{\mathrm{dyn}}\right)$ are compared with the theoretical value $\left(\mathrm{M}_{\mathrm{PS}}\right)$ determined 
Table 1. Parameters of Young Binary Systems

\begin{tabular}{lccccc}
\hline System & $\begin{array}{c}\mathrm{M}_{\text {dyn }} \\
\left(\mathrm{M}_{\odot}\right)\end{array}$ & $\begin{array}{c}\mathrm{M}_{\mathrm{PS}} \\
\left(\mathrm{M}_{\odot}\right)\end{array}$ & $\begin{array}{c}\left(\mathrm{M}_{A} / \mathrm{M}_{B}\right)_{\text {dyn }} \\
\left(\mathrm{M}_{\odot}\right)\end{array}$ & $\begin{array}{c}\left(\mathrm{M}_{A} / \mathrm{M}_{B}\right)_{\mathrm{PS}} \\
\left(\mathrm{M}_{\odot}\right)\end{array}$ & $\begin{array}{c}\mathrm{t}_{\mathrm{PS}} \\
\left(10^{6} \mathrm{yr}\right)\end{array}$ \\
\hline RS Cha & & & & & \\
A & $1.858 \pm 0.016$ & 1.88 & $1.02 \pm 0.02$ & $1.04 \pm 0.06$ & $5.0 \pm 0.3$ \\
B & $1.821 \pm 0.018$ & 1.80 & & & $4.3_{-0.6}^{+0.8}$
\end{tabular}

\section{EK Cep}

$\begin{array}{lccccc}\text { A } & 2.03 \pm 0.01 & 1.97 & 1.82 \pm 0.02 & 1.73_{-0.05}^{+0.15} & 20 \\ \text { B } & 1.12 \pm 0.01 & 1.14 & & & 20 \pm 4\end{array}$

\section{TY CrA}

$\begin{array}{lllllc}\mathrm{A} & 3.16 \pm 0.02 & 2.91 & 1.93 \pm 0.02 & 1.82_{-0.04}^{+0.11} & - \\ \mathrm{B} & 1.64 \pm 0.01 & 1.60 & & & 3.9_{-2.4}^{+3.6}\end{array}$

\section{BM Ori}

$\begin{array}{llllll}\mathrm{A} & 6.3 \pm 0.3 & 6.21 & 2.52 \pm 0.15 & 2.32_{-0.15}^{+0.15} & <0.1 \\ \mathrm{~B} & 2.5 \pm 0.1 & 2.68 & & & <0.1\end{array}$

V773 Tau

A

B

$1.53 \quad 1.32 \pm 0.06$

1.19

$1.29_{-0.23}^{+0.35}$

$4.1 \pm 0.9$

$2.8_{-1.0}^{+1.6}$

NTTS

162814
A
$1.26 \quad 1.09 \pm 0.07$
$1.11 \pm 0.07$
$5.1_{-2.1}^{+0.2}$
P 1540
A
1.71
$1.32 \pm 0.03$
$1.19 \pm 0.18$
$<0.1$
B
1.25
$0.2 \pm 0.1$

\section{GG Tau}

\begin{tabular}{cccc}
$\mathrm{Aa}$ & $1.28 \pm 0.07$ & 0.78 & $2.3 \pm 0.3$ \\
$\mathrm{Ab}$ & (total) & 0.54 & $1.5_{-0.5}^{+0.2}$ \\
$\mathrm{Ba}$ & & $<0.1$ & \\
$\mathrm{Bb}$ & & $\ll 0.1$ & \\
\hline
\end{tabular}




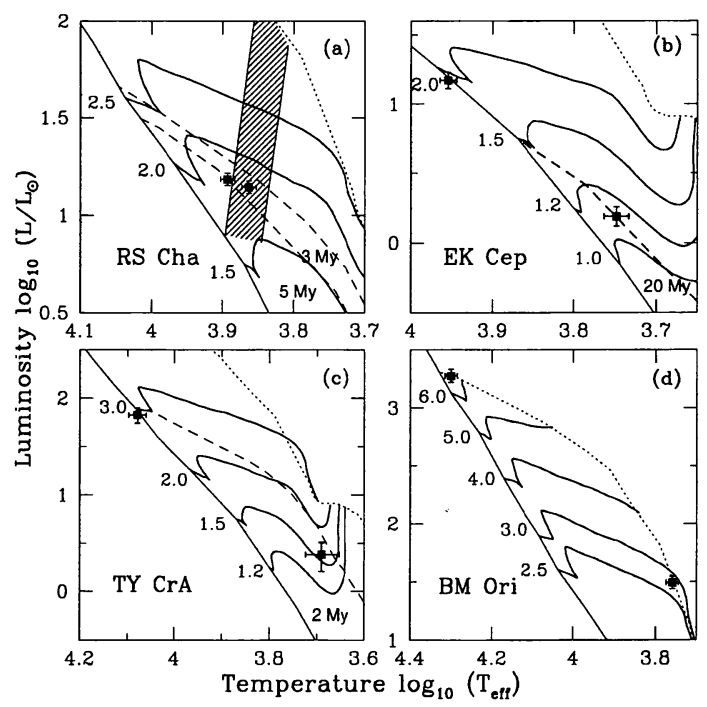

Figure 2. Tests of evolutionary tracks using PMS eclipsing binaries. In each panel the evolutionary tracks are shown by the solid lines, each labeled by the appropriate mass (in solar units). Dashed lines are isochrones, given in million years. The dotted line is the birthline computed with an accretion rate $\dot{M}=10^{-5} M_{\odot} \mathrm{yr}^{-1}$. In the case of RS Cha, the shaded area represents the instability strip for young stars. (From Palla \& Stahler 2000b)

from our set of tracks (Palla \& Stahler 1999). The next two columns list the mass ratios, both the observational ones $\left(\left(M_{A} / M_{B}\right)_{\text {dyn }}\right)$ and the derived values $\left(\left(M_{A} / M_{B}\right)_{\mathrm{PS}}\right)$. The last column gives our derived ages, with their uncertainties, for each component which appears to be PMS. The location of the four eclipsing systems RS Cha, EK Cep, TY Cra, and BM Ori is shown in Figure 2. Considering the mass estimates first, we note that the eclipsing binaries have small observational uncertainties (less than 1\%), BM Ori being the worst case (4\%). Thus, mass ratios in these cases are known with great precision. Not surprisingly, the error in the theoretical mass ratio is higher, varying between $2 \%$ and $9 \%$, but it is still rather small. Finally, the deviation from perfect agreement between the two estimates of the mass ratios amounts to $2 \%$ for RS Cha, $5 \%$ for EK Cep, $6 \%$ for TY CrA, and $10 \%$ for BM Ori which has the poorest dynamical mass estimate.

For double-lined, non-eclipsing spectroscopic binaries only the mass ratio is available. Figure 3 shows the position in the HR diagram of V773 Tau, NTTS $16284-2427, \mathrm{P} 1540$, and GG Tau. The uncertainty on the mass ratio is small, $2 \%$ to $6 \%$, whereas the theoretical values are less accurate. The two worst cases are V773 Tau and P1540 with uncertainties of about 20\%. As shown in Fig. 3, in both systems the secondary components are located on parts of the convective tracks that are closely spaced in mass. Thus, the error bars in $\left(\mathrm{L}, \mathrm{T}_{\text {eff }}\right)$ allow for rather different mass solutions. In the case of P1540, the error can be reduced by using as a further constraint the total luminosity of the system. Thus, only two 
solutions of the four possible pairs of $\left(\mathrm{L}, \mathrm{T}_{\text {eff }}\right)$ are allowed, and the uncertainty is limited to $18 \%$.

Even though the absolute errors on the theoretical mass ratios are worse than for the eclipsing binaries, the difference with the empirical ratios is quite small if we consider the nominal values given in Table 1 . The largest deviation of $10 \%$ is found for P1540, whereas the other cases are limited to $3 \%$.

Considering the results on the relative ages of the two components, we find again an overall agreement (cf. last column of Table 1). Of the eclipsing systems, only in RS Cha do both primary and secondary lie above the ZAMS and offer a reliable test for coevality. Here, we find a difference of $16 \%$ at the nominal position, which could amount to $40 \%$ considering the most unfavorable errors. The situation for the double-lined systems is not perfect, and differences of up to a factor of 1.5 (for P1540) are obtained. Evidently, age estimates are more sensitive to the uncertainties in the observed parameters.

The deviation in age of the binary components gauged by the isochrones is at most between 1 and $2 \times 10^{6}$ yr. Such a narrow range characterizes the wide binary systems studied by Hartigan et al. (1994) and Brandner \& Zinnecker (1996). Considering that the main star formation activity in a cluster lasts typically for about 3-4 million years (e.g. Palla \& Stahler 2000), such coevality suggests that most binaries are born in the same dense cores. Other dynamical processes, such as capture, are much less likely as possible formation mechanisms.

The tally of double-lined spectroscopic binaries is richer than that studied here. For example, PMS binaries with mass ratios very close to unity have not been included in Table 1, since they do not provide stringent tests to the goodness of the evolutionary tracks. Such systems include DQ Tau $(q=0.97)$, W134 $(q=1.04)$, and V826 Tau $(q=1.02)$. The theoretical masses match the empirical $q$-values to within $10 \%$.

Similarly, two other young eclipsing binaries have been discovered. One is GG Ori in the Orion OB1 association with a period of 6.6 days. Torres et al. (2000) have obtained precise masses (better than 1\%) for both components, which turn out to be quite similar $(q=1.002)$ and lie close to the ZAMS. Our mass estimate of $2.36 M_{\odot}$ for both components is very close to the empirical value of $2.34 M_{\odot}$. The location in the HR diagram indicates an age of $5 \times 10^{6} \mathrm{yr}$, consistent with the inferred age of the OB1 association (Brown et al. 1994), but greater ages cannot be excluded.

The second system, RXJ $0529.4+0041$, is potentially more interesting since both components are genuine PMS low-mass stars (Covino et al. 2000, this volume). Located in the Orion OB1a subgroup, the primary star is a weakline T Tauri star discovered by ROSAT. Both stars contain a high abundance of lithium, indicative of their relative youth. Preliminary analysis indicates that the orbit is essentially circular with a period of 3.0 days. The measured stellar masses are 1.30 and $0.95 M_{\odot}$, respectively, yielding a mass ratio of $q=0.73$. While the luminosity and effective temperature of the primary are well established, the secondary still suffers from a poor temperature determination. Using the values of Covino et al., we find a primary mass of $1.26 M_{\odot}$ in good agreement with the observed value, while the secondary has a lower value $\left(0.77 M_{\odot}\right)$. The two stars appear to be coeval with an age of $1 \times 10^{7} \mathrm{yr}$. 


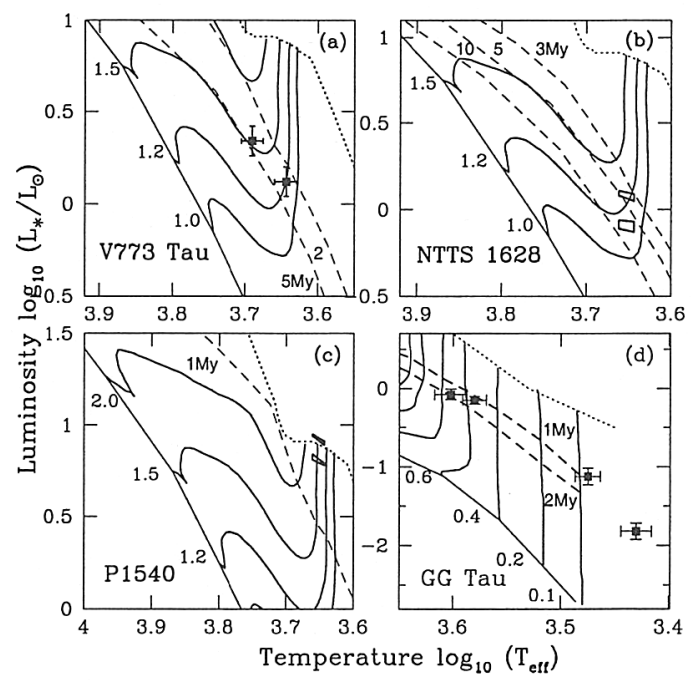

Figure 3. Tests of evolutionary tracks using PMS non-eclipsing spectroscopic binaries. The curves have the same meaning as in Fig. 2. The four systems are ordered from upper left to bottom right by decreasing mass of the primary component. For the GG Tau quadruple system, the faintest component lies below our minimum mass track $\left(0.1 M_{\odot}\right)$, in the brown dwarf regime. (From Palla \& Stahler 2000b)

An independent test of the accuracy of our set of pre-main-sequence tracks has been carried out by Simon et al. (2000, this volume), who have used mmwave interferometric data of circumstellar disks of a sample of single and binary $\mathrm{T}$ Tauri stars. On the assumption that the disk mass is small compared to the stellar mass and that the gas is in Keplerian rotation, the technique allows one to measure the mass dynamically. The accuracy of the inferred masses is estimated to be less than $10 \%$, over an interval in mass ranging from 2.0 to 0.5 $M_{\odot}$. We have repeated the analysis using the stellar parameters given by Simon and collaborators and found that our theoretical values agree to within $8 \%$ in all cases with a reliable dynamical mass estimate.

\section{Conclusions}

The favorable agreement between observed and theoretical masses of young binary systems that we have found is certainly encouranging, but there are still important tests that should be carried out to verify complete self-consistency. In particular, we note that with the exception of the two more massive components of the GG Tau system, all stars considered so far have masses near or above solar. However, the critical tests should be carried out on low-mass stars which are most sensitive to the uncertainties in the input physics. Also, very few systems are known with large values of the mass ratio. Because of the difficulty of detecting faint secondaries in the optical, most of the known spec- 
troscopic binaries are single-lined systems and the distribution of mass ratios in double-lined systems is artifically skewed toward unity. Mazeh et al. (2000, this volume) have shown that by observing single-lined binaries in the infrared, the cool, red secondaries can be effectively measured, thus transforming the system into a double-lined one. In this way, small mass ratios can be obtained.

A similar plea should be made to concentrate the observational efforts in the attempt to discover low-mass eclipsing binary systems. The recent case of RXJ 0529.4+0041 is quite encouraging and searches along the same directions should be pursued. Hopefully, soon we will be able to test the accuracy of PMS models in a much wider mass interval, thus increasing our level of confidence in the derivation of fundamental quantities such as the mass distribution and the star formation history of clusters and associations.

Acknowledgments. I wish to express my thanks to Bob Mathieu and Hans Zinnecker for the invitation to participate in such a memorable IAU conference. Much of the work presented here has been carried out in collaboration with S.W. Stahler.

\section{References}

Andersen, J. 1991, A\&A Rev., 3, 91

Basri, G., Marcy, G. W., \& Graham, J. R. 1996, ApJ, 458, 600

Bildsten, L. Brown, E. F., Matzner, C. D., \& Ushomirsky, G. 1997, ApJ, 482, 442

Bodenheimer, P. 1965, ApJ, 142, 451

Bonnell, I. A., Smith, K. W., Meyer, M. R., Tout, C. A., Folha, D. F. M., \& Emerson, J. P. 1998, MNRAS, 299, 1013

Brandner, W., \& Zinnecker, H. 1997, A\&A, 321, 220

Brown, A. G. A., de Geus, E. J., \& de Zeeuw, P. T. 1994, A\&A, 289, 101

Chabrier, G., \& Baraffe, I. 1995, ApJ, 451, L29

D'Antona, F., \& Mazzitelli, I. 1997, in Cool Stars in Clusters and Associations: magnetic activity and age indicators, ed. G. Micela et al., Mem. Soc. Astr. It., 68, 807

Delfosse, X., Forveille, T., Mayor, M., Burnet, M., \& Perrier, C. 1999, A\&A, 341, L63

Hartigan, P., Strom, K. M., \& Strom, S. E. 1994, ApJ, 427, 961

Hillenbrand, L. A. 1997, AJ, 113, 1733

Hillenbrand, L. A., Massey, P., Strom, S. E., \& Merrill, K. M. 1993, AJ, 106, 1906

Luhman, K. L. 1999, ApJ, 525, 466

Marconi, M., \& Palla, F. 1998, ApJ, 507, L141

Marconi, M., Ripepi, V., Alcalá, J. M., Covino, E., Palla, F., \& Terranegra, L. 2000, A\&A, 355, L35

Martín, E. L., \& Claret, A. 1996, A\&A, 306, 408

Massey, P., \& Hunter, D. A. 1998, ApJ, 493, 180 
Massey, P., Johnson, K. E., \& DeGioia-Eastwood, K. 1995, ApJ, 454, 151

McNamara, B. J. 1976, AJ, 81, 845

Palla, F., \& Stahler, S. W. 1999, ApJ, 525, 772

Palla, F., \& Stahler, S. W. 2000a, ApJ, 540, 255

Palla, F., \& Stahler, S. W. 2000b, ApJ, in press

Pinsonneault, M. H., Stauffer, J., Soderblom, D. R., King, J. R., \& Hanson, R. B. 1998, ApJ, 504, 170

Rebolo, R., Martín, E. L., Basri, G., Marcy, G. W., \& Zapatero Osorio, M. R. 1996, ApJ, 469, L53

Torres, G., Lacy, G. H. S., Claret, A., \& Sabby, J. A. 2000, AJ, in press

van den Ancker, M. E., de Winter, D., \& Tjin A Djie, H. R. E. 1998, A\&A, 330, 145

van Leeuwen, F., \& Ruiz, H. C. S. 1997, A\&A, 402, 689

Ventura, P., Zeppieri, A., Mazzitelli, I., \& D'Antona, F. 1998, A\&A, 331, 1011

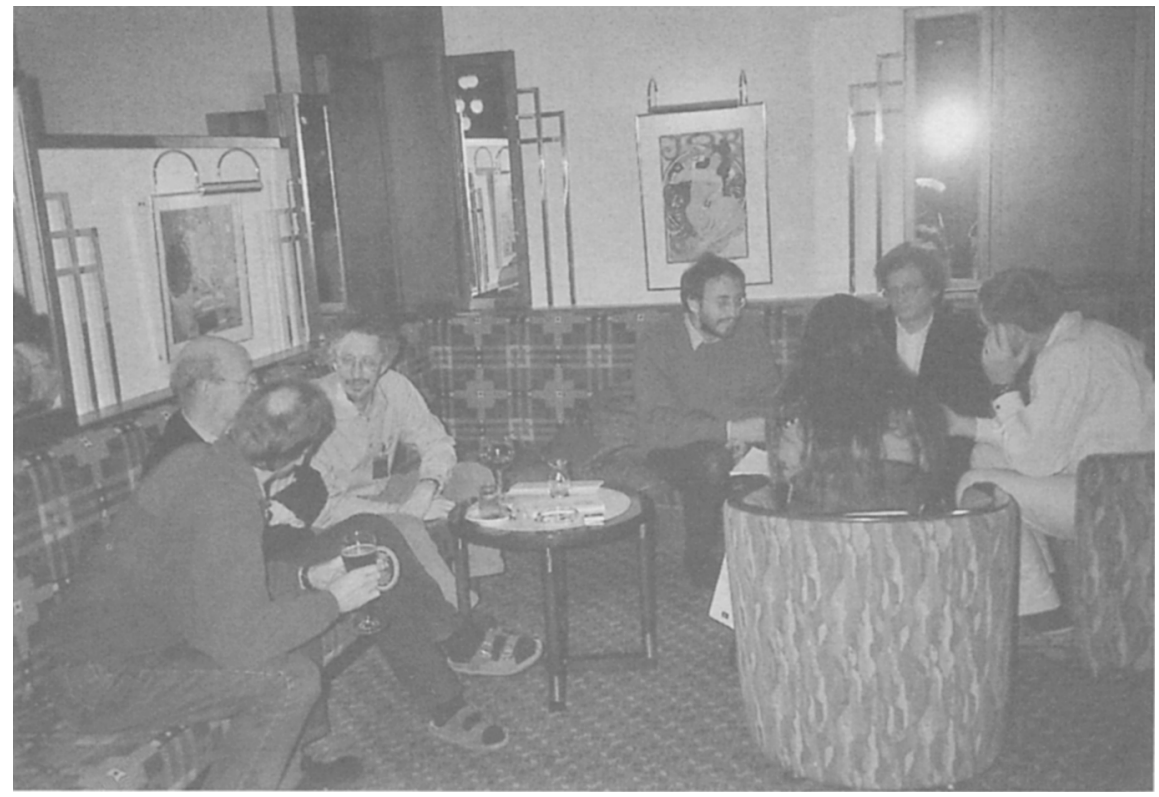

Far end: Günther Wuchterl, Francesco Palla, Pavel Kroupa and Isabelle Baraffe 

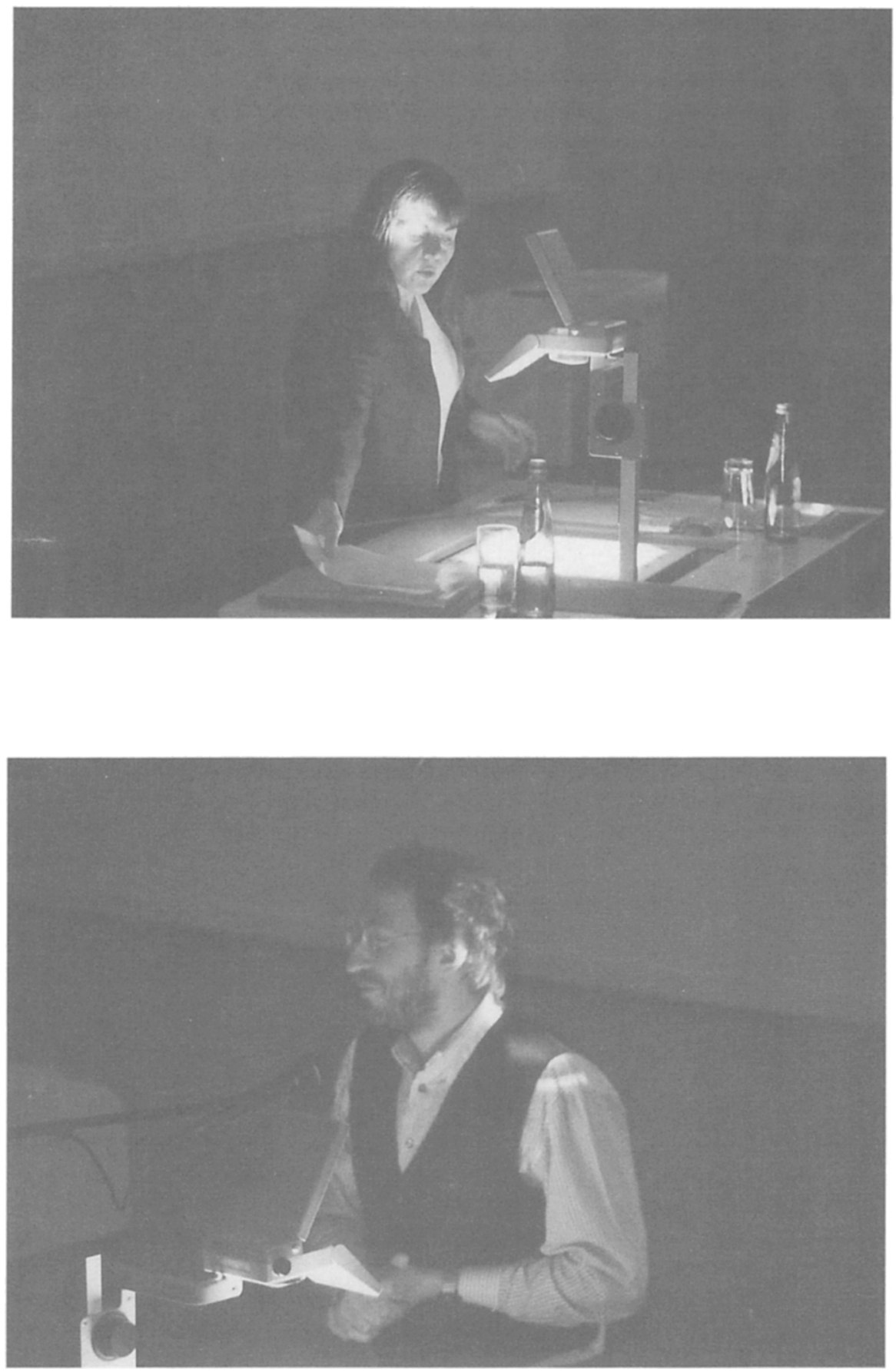

The big debate between Isabelle Baraffe (top) and Günther Wuchterl (bottom) 